\title{
A SIMPLE FABRICATION METHOD FOR DOUBLY REENTRANT OMNIPHOBIC SURFACES VIA STRESS INDUCED BENDING
}

\author{
Kyle L. Wilke ${ }^{1 *}$, Manuel Garcia ${ }^{l}$, Daniel J. Preston ${ }^{l}$, and Evelyn N. Wang ${ }^{l}$
}

${ }^{1}$ Massachusetts Institute of Technology, Cambridge, Massachusetts, USA

\begin{abstract}
We developed omniphobic, doubly reentrant surfaces fabricated with a simple method suitable for use with traditional microfabrication processes. Intrinsic stresses in deposited layers of silicon nitride induced bending of a singly reentrant microstructure, creating the doubly reentrant geometry. Due to the use of standard microfabrication processes, this approach may be extended to a variety of materials and feature sizes, increasing the viability of applying omniphobic doubly reentrant structures for use in areas such as superomniphobicity, anti-corrosion, heat transfer enhancement, and drag reduction.
\end{abstract}

\section{INTRODUCTION}

Doubly reentrant microstructures enable omniphobic surfaces that repel even completely wetting (i.e., low surface tension) fluids without the use of low-surface-energy coatings [1-3]. Such surfaces have use in a range of applications such as anti-fouling [4], chemical shielding [5], heat transfer enhancement [6], drag reduction [7], selfcleaning [8], and water purification [9]. In order to achieve omniphobicity, reentrant microstructures with characteristic length smaller than the liquids capillary length are required [1]. The level of reentrance necessary depends on the fluid's intrinsic contact angle, $\theta$, with the surface material (Figure 1a). The black arrow in the figure represents the contact line force. In order for liquid to be repelled, a component of the contact line force must act in the vertical direction. Therefore, a regular microstructure without reentrance only repels liquids with $\theta>90^{\circ}$. This large intrinsic contact angle is not possible for many liquids on solid surfaces or requires chemical modification for many others. Adding reentrant geometry can reduce the necessary contact angle to below $90^{\circ}$. However, for a small $\theta$, the vertical component of the contact line force is also small, making it difficult to repel highly wetting liquids. Therefore, to repel all liquids, including those with a small contact angle, a large degree of reentrance is required (i.e., doubly reentrant), which has been difficult to fabricate at the necessary characteristic length scales (100s of microns or smaller). Due to these difficulties, the fabrication of doubly reentrant surfaces has been limited in materials used, i.e., specialized polymers [2], or required complex fabrication techniques, i.e., high temperature

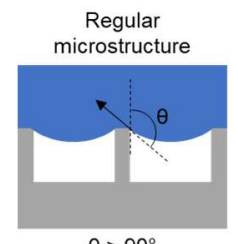

$\theta>90^{\circ}$

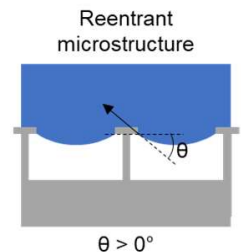

$\theta>0^{\circ}$

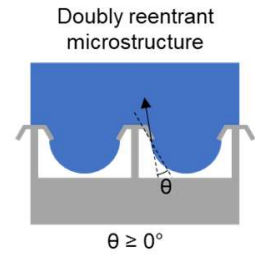

$\theta \geq 0^{\circ}$
Figure 1: Types of microstructures along with corresponding intrinsic contact angle, $\theta$, for which the structure can practically repel liquid. The black arrow represents the contact line force that prevents liquid from entering the structure, which must act in the vertical direction in order to repel the liquid. To successfully repel liquids with low contact angle, doubly reentrant structures are necessary. oxidation of silicon (not suitable for other materials) and multiple etch steps [1].

In this work, we developed a simple fabrication method using traditional microfabrication processes and demonstrated omniphobicity of the surface. The method relies on intrinsic stresses of deposited materials to induce bending of singly reentrant structures to create doubly reentrant geometry. Because this fabrication technique is based on singly reentrant surfaces, for which robust, scalable processes are already well-developed [10-12], it enables simple fabrication of doubly reentrant structures for a wide variety of materials.

\section{RESULTS}

The level of double reentrance, $\alpha$, influences the component of contact line force that acts in the vertical direction, $F_{y}$, which prevents liquid from entering the structures and maintains omniphobicity. Figure 2 shows the vertical component of $F$, for different $\theta$ and $\alpha$, normalized by the theoretical maximum that occurs when the force is completely vertical, $F_{y, \max }$, and is defined as:

$$
F_{\text {norm }}=\frac{F_{y}}{F_{y, \max }}=\frac{P \gamma \sin (\theta+\alpha)}{P \gamma}
$$

where $P$ is the length of the contact line (determined by microstructure geometry), and $\gamma$ is the surface tension of the liquid. If the sum of $\theta$ and $\alpha$ is greater than $90^{\circ}$, then $F_{\text {norm }}=1$ due to the fact that a maximum occurs in $F_{y}$ for a contact angle below the intrinsic value, which is still a stable state. This is why the curves for different $\theta$ reach a stable value of 1 with increasing $\alpha$. This figure highlights a number of key points. First, in order to produce adequate repellency of fluids with $\theta=0^{\circ}, \alpha$ must be larger than 0 . Second, the repellency force increases with $\alpha$, which creates a more

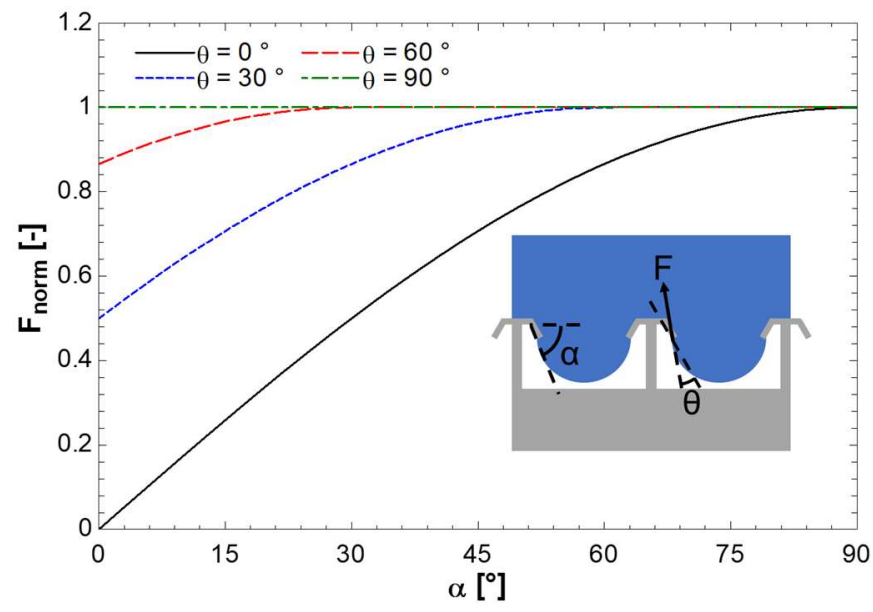

Figure 2: The normalized vertical component of the contact line force for different contact angle, $\theta$, and level of reentrance, $\alpha$. For a perfectly wetting liquid, $\alpha$ must be greater than $0^{\circ}$ for the surface to have repellency. The maximum is reached when $\theta+\alpha=90^{\circ}$. 
robust omniphobicity, but reaches its maximum value when $\theta+\alpha=$ $90^{\circ}$. We note that though the maximum repellency is reached at $\theta+$ $\alpha=90^{\circ}$, a lesser value of $\alpha$ may still produce adequate repellency.

Figure 3 shows our fabrication process. Thin films of silicon nitride with intrinsic stresses were deposited on the polished silicon wafer with an STS PECVD system, which allowed stresses in the deposited material to be controlled (i). Next, features were defined with photolithography (ii). A $2.5 \mu \mathrm{m}$ thick layer of Microposit S1823 was spin coated on the wafer and exposed using an MLA150 Maskless Aligner. The resist was developed in CD-26 for 90 seconds. Structures were then etched with DRIE (iii). An STS ICP RIE was used to etch the silicon nitride layers. Subsequently, an SPTS Rapier DRIE was used to etch the underlying silicon. Finally, an isotropic silicon etch was used to undercut the thin films of silicon nitride (iv). Both $\mathrm{XeF}_{2}$ and $\mathrm{SF}_{6}$ were successfully used for the isotropic etch due to the etch selectivity between silicon nitride and silicon. Due to the intrinsic stresses in the nitride films, tunable bending (based on film thickness, stress, and undercut distance) occurred and formed the doubly reentrant surface.
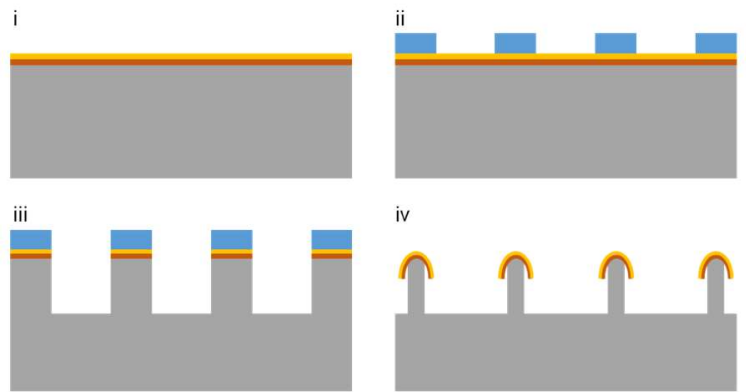

KEY:

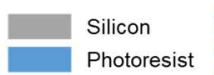

$+$

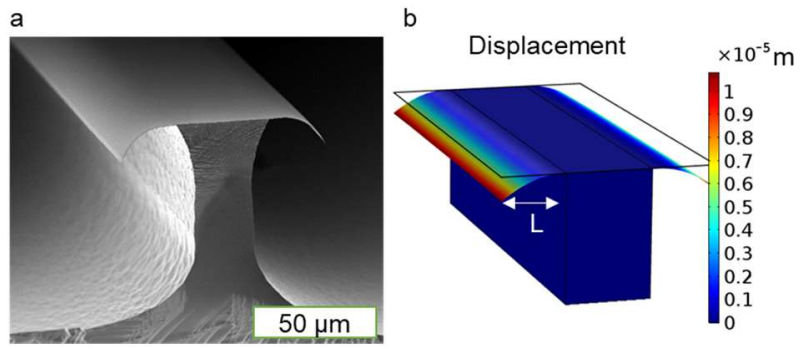

Figure 4: a: Cross-section SEM of the fabricated doubly reentrant $\left(\alpha=62^{\circ}\right)$ stripe-textured hoodoo surface. $b$ : COMSOL model used to predict surface bending based on stresses and film thicknesses. This model determined the necessary stresses and thicknesses to realize a doubly reentrant surface with significant bending. For silicon nitride, this required the ratio of the length of the feature undercut, $L$, to the combined film thicknesses to be between 10 and 100, where 100 is shown in this figure.

We characterized the apparent advancing and receding contact angle of the fabricated surface for a variety of liquids using the experimental setup depicted in Figure 5. A syringe was used to add and remove liquid from a droplet on the surface. A collimated light source behind the droplet illuminated it while a DSLR camera recorded video as the droplet grew and shrunk. The video was then used to extract the advancing and receding contact angles in ImageJ.

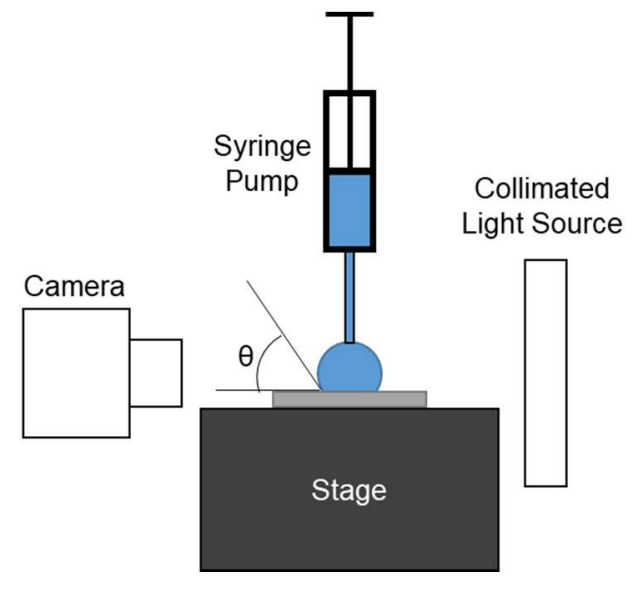

Figure 5: Schematic of experimental setup for characterizing contact angle. A syringe is used to add and remove liquid from a droplet on the surface, creating an advancing and receding contact line, respectively.

Images of the apparent advancing contact angle of both water, with a surface tension of $72.8 \mathrm{mN} / \mathrm{m}$ and $\mathrm{FC} 40$, a common refrigerant with a very low surface tension of $16 \mathrm{mN} / \mathrm{m}$, are shown in Figure 6. Due to the double reentrant structures and surface omniphobicity, both fluids were repelled regardless of the liquid surface tension. 


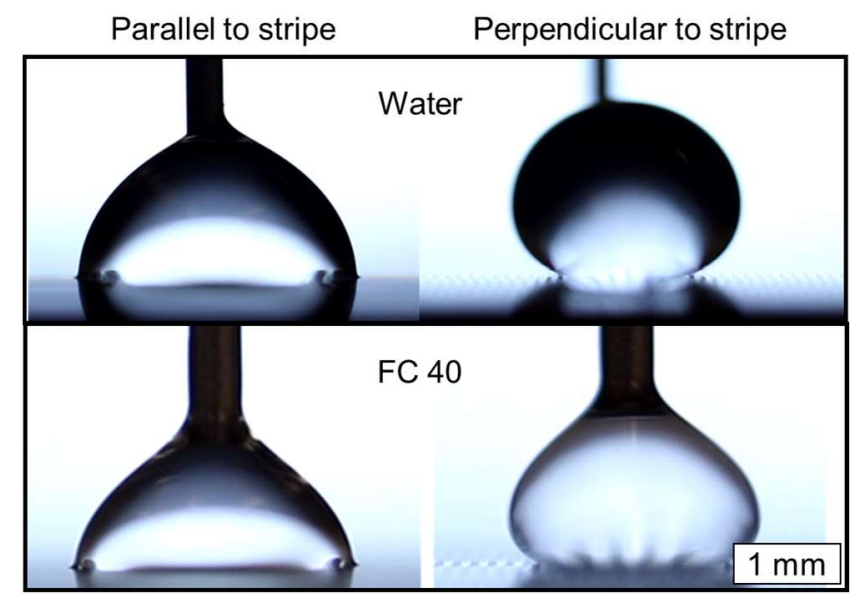

Figure 6: Images of advancing contact angle on the stripe-textured hoodoo surface, both parallel and perpendicular to the stripe for both water and FC 40. Both fluids were repelled due to the surface omniphobicity.

Advancing and receding contact angle measurements were conducted for liquids with a wide range of surface tensions (FC-40, ethanol, isopropanol, ethylene glycol, and water) in Figure 7. The observed contact angles agree well with theoretical values [11]. For the stripe-textured hoodoo surfaces the advancing contact angle

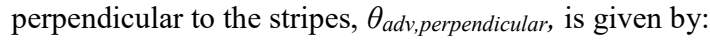

$$
\cos \theta_{\text {adv,perpendicular }}=-1
$$

which gives an expected value of $180^{\circ}$. The observed values are slightly lower due to vibrations in the laboratory causing the droplets to partially relax. The advancing contact angle parallel to the stripes, $\theta_{\text {adv, parallel, }}$ is given by:

$$
\cos \theta_{\text {adv,parallel }}=r \phi \cos \theta-(1-\phi)
$$

where $r$ is the roughness of the wetted solid (taken as 1 in this work due to the thin nitride films), and $\phi$ is the local areal solid fraction of the advancing droplet, 0.5 for the surface demonstrated here. This gives a predicted value of $90^{\circ}$, similar to the experimentally observed values. Finally, the receding contact angle in both the parallel and perpendicular directions, $\theta_{\text {rec }}$, is given by:

$$
\cos \theta_{\text {rec }}=r \phi \cos \theta-(1-\phi)
$$

For the stripe-textured surface used here the predicted receding angle is the same as the advancing angle parallel to the stripes, leading to a low contact angle hysteresis, which is necessary for liquid shedding. Furthermore, due to the doubly reentrant geometry the fluid properties do not appear in equations 2 through 4 . Therefore, measured contact angles in Figure 7 are independent of the liquid contacting the surface, which agrees well with observed values. The advancing contact angles on a flat silicon nitride surface are also shown. Despite the nearly perfectly wetting liquids used, they were still successfully repelled.

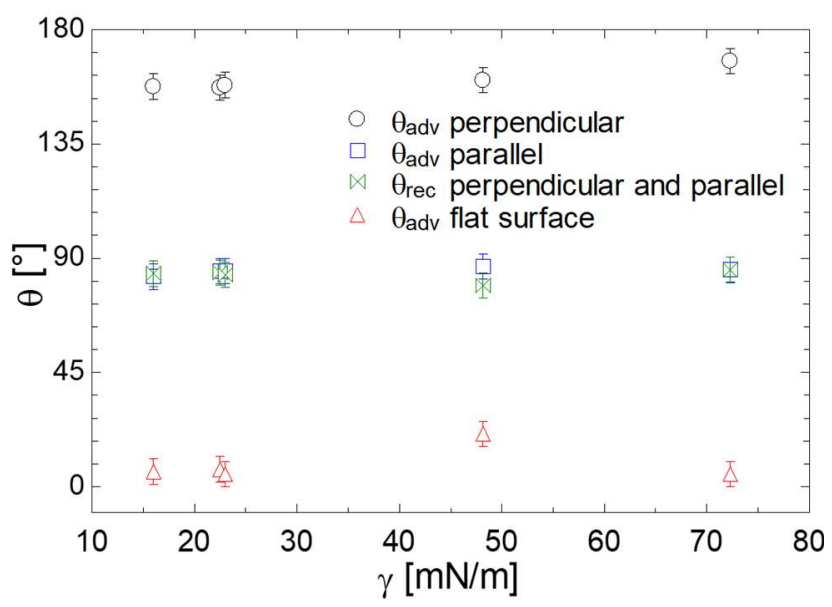

Figure 7: Apparent advancing and receding contact angle of liquids with different surface tension, $\gamma$, both parallel and perpendicular to the stripe. Receding angle was the same in both directions. Advancing contact angle on a flat silicon nitride surface is also shown. All fluids were repelled, highlighting the omniphobicity, which can only be achieved with doubly reentrant geometry.

We are currently working to demonstrate different material sets and types of reentrant geometry (other than stripe-textured). Though silicon nitride was used in this work, other materials also have controllable stresses (including metals, oxides, nitrides, and polymers) and are potential material choices. Table 1 lists a variety of materials and residual stresses observed in literature after growth/deposition with different techniques such as chemical vapor deposition (CVD), plasma-enhanced chemical vapor deposition (PECVD), magnetron sputtering, spin coating, and atomic layer deposition (ALD). Although this incomplete list represents candidate materials, further research should be done to determine necessary deposition parameters, how stresses in the films may evolve with time, as well as what $\alpha$ may be achieved. Furthermore, in order to fabricate the reentrant geometry, etch selectivity between the deposited material and the underlying substrate is necessary, which may limit material choices.

Table 1: Possible materials, deposition techniques, and residual stresses reported in the literature. A negative stress is compressive.

\begin{tabular}{|c|c|c|c|}
\hline Material & $\begin{array}{c}\text { Deposition } \\
\text { Technique }\end{array}$ & $\begin{array}{c}\text { Residual } \\
\text { Stress [MPa] }\end{array}$ & Reference \\
\hline Silicon nitride & PECVD & 300 to -600 & {$[13]$} \\
\hline Metals & Sputtering & 1000 to -2000 & {$[14]$} \\
\hline Parylene & CVD & 50 to 20 & {$[15]$} \\
\hline Polyimide & Spin coat & 30 & {$[16]$} \\
\hline Aluminum Oxide & ALD & 500 to 200 & {$[17]$} \\
\hline
\end{tabular}

\section{CONCLUSIONS}

This fabrication procedure simplifies the ability to create doubly reentrant surfaces. Stress induced bending of singly reentrant geometry into doubly reentrant geometry was achieved by relying on intrinsic stresses of deposited silicon nitride films. Finite element analysis (FEA) was used to predict the necessary stresses, film thicknesses, and undercut length, $L$, to achieve a high level of reentrance. A stripe-textured hoodoo surface was fabricated and 
good agreement was observed with the FEA model. Due to the high level of reentrance achieved $\left(\alpha=62^{\circ}\right)$, the fabricated surface was able to repel all tested liquids with surface tension ranging from 16 to $72.8 \mathrm{mN} / \mathrm{m}$, including liquids with very small intrinsic contact angle on silicon nitride. Furthermore, the observed contact angles matched well with predicted values based on previously developed models. Due to the use of standard microfabrication processes, the process developed here expands the potential materials sets, and widens the potential application of doubly reentrant omniphobic surfaces.

\section{ACKNOWLEDGEMENTS}

This work was supported by the Cooperative Agreement between the Masdar Institute of Science and Technology (Masdar Institute), Abu Dhabi, UAE and the Massachusetts Institute of Technology (MIT), Cambridge, MA, USA - Reference 02/MI/MIT/CP/11/07633/GEN/G/00. This work was performed in part at the Center for Nanoscale Systems (CNS), a member of the National Nanotechnology Infrastructure Network (NNIN), which is supported by the National Science Foundation under NSF award no. ECS-0335765. CNS is part of Harvard University.

\section{REFERENCES}

[1] T. Liu and C.-J. Kim, "Turning a surface superrepellent even to completely wetting liquids". Science, 346(6213), (2014).

[2] J. Choi, W. Jo, S.Y. Lee, Y.S. Jung, S.-H. Kim, and H.-T. Kim, "Flexible and Robust Superomniphobic Surfaces Created by Localized Photofluidization of Azopolymer Pillars". ACS nano, 11(8): p. 7821-7828, (2017).

[3] R. Hensel, R. Helbig, S. Aland, A. Voigt, C. Neinhuis, and C. Werner, "Tunable nano-replication to explore the omniphobic characteristics of springtail skin". NPG Asia Materials, 5(2): p. e37, (2013).

[4] D.C. Leslie, et al., "A bioinspired omniphobic surface coating on medical devices prevents thrombosis and biofouling". Nature biotechnology, 32(11): p. 1134-1140, (2014).

[5] S. Pan, A.K. Kota, J.M. Mabry, and A. Tuteja, "Superomniphobic surfaces for effective chemical shielding". Journal of the American Chemical Society, 135(2): p. 578581, (2012).

[6] T. Liu and C.-J. Kim. Doubly re-entrant cavities to sustain boiling nucleation in FC-72. in Micro Electro Mechanical Systems (MEMS), 2015 28th IEEE International Conference on. 2015: IEEE.

[7] C.-H. Choi and C.-J. Kim, "Large slip of aqueous liquid flow over a nanoengineered superhydrophobic surface". Physical review letters, 96(6): p. 066001, (2006).

[8] Y. Lu, S. Sathasivam, J. Song, C.R. Crick, C.J. Carmalt, and I.P. Parkin, "Robust self-cleaning surfaces that function when exposed to either air or oil". Science, 347(6226): p. 11321135, (2015).

[9] J. Lee, C. Boo, W.-H. Ryu, A.D. Taylor, and M. Elimelech, "Development of omniphobic desalination membranes using a charged electrospun nanofiber scaffold". ACS Applied Materials \& Interfaces, 8(17): p. 11154-11161, (2016).

[10] A. Tuteja, W. Choi, J.M. Mabry, G.H. McKinley, and R.E. Cohen, "Robust omniphobic surfaces". Proceedings of the National Academy of Sciences, 105(47): p. 18200-18205, (2008).

[11] W. Choi, A. Tuteja, J.M. Mabry, R.E. Cohen, and G.H. McKinley, "A modified Cassie-Baxter relationship to explain contact angle hysteresis and anisotropy on non-wetting textured surfaces". Journal of colloid and interface science, 339(1): p. 208-216, (2009).
[12] A. Tuteja, W. Choi, M. Ma, J.M. Mabry, S.A. Mazzella, G.C. Rutledge, G.H. McKinley, and R.E. Cohen, "Designing superoleophobic surfaces". Science, 318(5856): p. 1618-1622, (2007).

[13] M.P. Martyniuk, J. Antoszewski, C.A. Musca, J.M. Dell, and L. Faraone. Determination of residual stress in lowtemperature PECVD silicon nitride thin films. in Device and Process Technologies for MEMS, Microelectronics, and Photonics III. 2004: International Society for Optics and Photonics.

[14] D. Hoffman and J.A. Thornton, "Effects of substrate orientation and rotation on internal stresses in sputtered metal films". Journal of Vacuum Science and Technology, 16(2): p. 134-137, (1979).

[15] T.A. Harder, T.-J. Yao, Q. He, C.-Y. Shih, and Y.-C. Tai. Residual stress in thin-film parylene-C. in Micro Electro Mechanical Systems, 2002. The Fifteenth IEEE International Conference on. 2002: IEEE.

[16] M.G. Allen, M. Mehregany, R.T. Howe, and S.D. Senturia, "Microfabricated structures for the insitu measurement of residual stress, young's modulus, and ultimate strain of thin films". Applied physics letters, 51(4): p. 241-243, (1987).

[17] O.M. Ylivaara, et al., "Aluminum oxide from trimethylaluminum and water by atomic layer deposition: The temperature dependence of residual stress, elastic modulus, hardness and adhesion". Thin Solid Films, 552: p. 124-135, (2014).

CONTACT

*K.L. Wilke, tel: +1-608-620-4977; klwilke@mit.edu 